\title{
Modelling a type-2 fuzzy inventory system considering items with imperfect quality and shortage backlogging
}

\author{
RAVI SHANKAR KUMAR \\ National Institute of Technology Agartala, Agartala 799046, India \\ e-mail: ravikhushi412@yahoo.co.in
}

MS received 2 December 2017; revised 31 January 2018; accepted 1 February 2018; published online 24 August 2018

\begin{abstract}
In this paper, an inventory model considering items with imperfect quality is developed in fuzzy environment, wherein shortages are allowed and are backlogged. In order to detect the items with imperfect quality, all items are screened before they are sent for consumption, and all imperfect quality items are sold at discounted price, called salvage value. In view of the fact that demand may not be predicted precisely, because it depends upon many uncertain and perturbing market activities, it is assumed to be a type- 2 fuzzy variable. Quantity of imperfect quality items in the received lot may not be predicted precisely; hence percentage of imperfect quality items is also considered as a type-2 fuzzy variable. In this regard, this study developed a defuzzification method of type- 2 fuzzy variable pertaining to interval approximation. The mathematical model is analysed to find closed form formulae of order quantity and backlogging quantity. Finally, the proposed methodology and model are testified on numerical examples, and sensitivity of decision variables is examined and discussed to underline the managerial insights as well as to establish the robustness of the mathematical model.
\end{abstract}

Keyword. Inventory; imperfect quality item; screening; shortage backlogging; type-2 fuzzy set; interval approximation.

\section{Introduction}

Demand is one of the most important factors because due to it a business and hence an inventory management problem exists [1, 2]. Thus, proper estimation of demand is an inevitable but challenging task that depends upon many perturbing and uncertain activities. Demand estimation process, in general, depends upon previous data records and/or anticipation of forthcoming scenarios. Many times due to imperfect documentation, carelessness and disappearance of sources, previous data records do not carry full information. This ill-known data and lack of information result in an imprecise boundary of the demand, and it can be represented by a fuzzy variable. Fuzzy inventory systems considering fuzzy and fuzzy random demands have been widely addressed (see, [3]). However, many times it is not possible to express the demand quantity with a fixed grade of membership. Let us suppose that 10 experts are assigned to predict the demand of motor parts for forthcoming year. Each expert opinion is vague due to said reasons, and is presented by a fuzzy set with grade of membership. Out of 10 experts, consider that three say the grade of membership of 100 units is 0.3 , four experts say it is 0.4 , two experts say it is 0.2 and two experts say it is 0.2 . Here vagueness in grade of membership is also inherited.
This vagueness can be expressed in terms of a fuzzy set in the domain $[0,1]$. Thus, vagueness in demand as well as in its grade of membership has been noticed. This type of demand can be modelled with the help of type- 2 fuzzy variables. This study is intended to develop an inventory model by considering type- 2 fuzzy demand and type- 2 fuzzy imperfect quality of items. In this process, a defuzzification method, based on interval approximation, of type-2 fuzzy variable has been proposed.

An item whose quality is not $100 \%$ perfect (i.e., partially defective) due to manhandling and/or machine error is termed here as imperfect quality of item. Imperfect quality of item can be used if one agrees to compromise with the quality. There are so many reasons such as defective production process, equipment failure, natural disasters and damage or breakage in transit, due to which the lot size produced/received may contain a fraction of imperfect quality of item. Rosenblatt and Lee [4] assumed that the production process may deteriorate due to time factor and continuous use; consequently, produced items are of imperfect quality. Later, Salameh and Jaber [5] proposed an economic order quantity (EOQ) model by considering screening facility of item that is accomplished before selling, and they found imperfect quality of items sold in a single batch at some discounted price. Wee et al [6] and Eroglu and Ozdemir [7] 
extended [5] by allowing shortages. Eroglu and Ozdemir [7] considered that defective items are a mixture of imperfect items and scraps, and scraps do not have any value; hence, they are thrown away instantly from the system. After that many authors have enhanced/extended [5-7] in several aspects such as inspection error, quantity discount and so on; some recommendable ones among them are [8-11], etc. Wang et al [12] extended [6] and [11] by considering partial backlogging of shortage, and employed screening constraints. Rezaei [13] considered that inspection of all items is a costly process and is not always a good decision; hence, he proposed a model by considering sampling inspection plan. Taleizadeh et al [14] considered reparation of imperfect quality products; for this, imperfect quality products are immediately sent to a local repair shop. Khan et al [15] derived an economic production quantity (EPQ) formula for vendor-buyer supply chain by considering screening error.

Fuzzy set theory relaxes the essentiality of carrying the full information concerning previous data records and provides flexibility in forecasted value, and is a suitable way to present the key components of inventory problem. Due to this, fuzzy set theory and its variants are widely applied in inventory modelling problems. A comprehensive literature survey on fuzzy inventory models was carried out by Shekarian et al [3]. A brief literature review, focusing on fuzzy demand and fuzzy defective items, is presented here by finding out the research gap. Yao et al [16] extended the classical EOQ model by assuming that demand rate is a fuzzy number, and employed the extension principle to obtain the fuzzy total cost function. Chang [17] extended [5] in fuzzy environment by considering demand rate and fraction of imperfect quality items as fuzzy numbers. Bjork [18] extended the classical EOQ model with backorder by assuming that demand and lead time are fuzzy numbers. Liu and Zheng [19] extended [6] by considering fuzzy demand and imperfect quality of items, and employed GMIR (graded mean integration representation) and centroid methods to encapsulate the model. Kumar and Goswami [20] extended distribution-free continuous review inventory system in fuzzy random environment, and employed fuzzy random renewal reward theorem to find the average cost function. Kumar et al [21] developed an integrated production-inventory for vendor-buyer integrated supply chain by considering fuzzy random demand rate. The continuous review and periodic review inventory systems and their variants were extended in fuzzy environment by considering fuzzy/fuzzy random demand by [22-30].

Type- 2 fuzzy sets have been widely applied in many areas such as decision making, solving fuzzy relation equations and pre-processing of data ([31]). However, as an evidence of afore-mentioned literature review and to the best of my knowledge, no inventory model has been proposed in type-2 fuzzy environment. Thus, the main objective of this research is to formulate a type-2 fuzzy inventory model. Basically, the present paper extends [6] in fuzzy environment by considering demand rate and fraction of imperfect quality of item as interval type- 2 fuzzy sets
(IT2FSs). In this process, a new method of de-fuzzification of IT2FS is proposed and applied to the model. The mathematical model is analysed to find closed form formulae of order quantity and backordered quantity. Comparative study with other existing models has been made, and the model is deduced to [6] and [5] under some restrictions. The rest of the paper is arranged as follows. Section 2 is the methodology section, wherein basics and then the proposed de-fuzzification method are presented. Notations and assumptions are listed in section 3, which are used in the entire paper. In section 4, the model is mathematically formulated, and optimal decision policy in closed form is also derived. Section 5 deduces the existing models from the proposed one. Section 6 has a numerical example, wherein the proposed methodology and model formulation are justified with an illustrative example and sensitivity analysis by changing the fuzzy parameters' value. The discussion is ended with concluding remarks and potential future research direction.

\section{Methodology}

This section provides a brief introduction to type- 2 fuzzy set, and then proposes a new method of de-fuzzification. For a detailed study on type- 2 fuzzy sets, one can go through [32-35].

\subsection{Preliminaries}

Mizumoto and Tanaka [36] proposed algebraic operations and properties of membership functions of a type-2 fuzzy set with the help of extension principle. Karnik and Mendel [31] and Chen [35], respectively, proposed the centriod and signed distance methods of de-fuzzification of type-2 fuzzy set. Here, only the basic definitions and results that are used throughout the paper are discussed.

2.1a IT2FS [34, 35]: Let $X \subset \mathcal{R}$ (real numbers) be a finite non-empty set, called universal set, and let $\operatorname{Int}([0,1])$ be the collection of all closed subintervals of $[0,1]$. An IT2FS $\tilde{A}$ in $X$ is defined as $\tilde{A}=\left\{\left(x, \mu_{\tilde{A}}(x)\right): x \in X\right\}$, where $\mu_{\tilde{A}}: X-$ $\rightarrow \operatorname{Int}([0,1])$ is defined as $\mu_{\tilde{A}}(x)=\left[\mu_{\tilde{A}}^{-}, \mu_{\tilde{A}}^{+}\right], 0 \leq \mu_{\tilde{A}}^{-} \leq$ $\mu_{\tilde{A}}^{+} \leq 1$, i.e., grade of membership of $x \in \tilde{A}$ is an interval $\left[\mu_{\tilde{A}}^{-}, \mu_{\tilde{A}}^{+}\right]$.

2.1b Interval type-2 trapezoidal fuzzy set (IT2TrFS) [35]: An interval type-2 trapezoidal fuzzy set (IT2TrFS) is a IT2FS $\tilde{A}=\left[\tilde{A}^{L}, \tilde{A}^{U}\right]=\left[\left(a_{1}^{L}, a_{2}^{L}, a_{3}^{L}, a_{4}^{L} ; h_{A}^{L}\right),\left(a_{1}^{U}, a_{2}^{U}, a_{3}^{U}\right.\right.$, $\left.\left.a_{4}^{U} ; h_{A}^{U}\right)\right]$, where $\tilde{A}^{L}$ and $\tilde{A}^{U}$ are generalized trapezoidal fuzzy numbers and $\tilde{A}^{L} \subseteq \tilde{A}^{U} . \tilde{A}^{L}$ and $\tilde{A}^{U}$ are defined by lower membership function (LMF) $\mu_{\tilde{A}}^{L}$ and upper membership function (UMF) $\mu_{\tilde{A}}^{U}$ as follows: 


$$
\mu_{\tilde{A}}^{L}=\left\{\begin{array}{cc}
h_{A}^{L}\left(\frac{x-a_{1}^{L}}{a_{2}^{L}-a_{1}^{L}}\right), & a_{1}^{L} \leq x \leq a_{2}^{L} ; \\
h_{A}^{L}, & a_{2}^{L} \leq x \leq a_{3}^{L} ; \\
h_{A}^{L}\left(\frac{a_{4}^{L}-x}{a_{4}^{L}-a_{3}^{L}}\right), & a_{3}^{L} \leq x \leq a_{4}^{L} ; \\
0, & \text { otherwise; }
\end{array}\right.
$$

and

$$
\mu_{\tilde{A}}^{U}=\left\{\begin{array}{cc}
h_{A}^{U}\left(\frac{x-a_{1}^{U}}{a_{2}^{U}-a_{1}^{U}}\right), & a_{1}^{U} \leq x \leq a_{2}^{U} ; \\
h_{A}^{U}, & a_{2}^{U} \leq x \leq a_{3}^{U} ; \\
h_{A}^{U}\left(\frac{a_{4}^{U}-x}{a_{4}^{U}-a_{3}^{U}}\right), & a_{3}^{U} \leq x \leq a_{4}^{U} ; \\
0, & \text { otherwise. }
\end{array}\right.
$$

2.1c Arithmetic operations on IT2TrFS [35]: Let $\tilde{A}=$ $\left[\tilde{A}^{L}, \tilde{A}^{U}\right]=\left[\left(a_{1}^{L}, a_{2}^{L}, a_{3}^{L}, a_{4}^{L} ; h_{A}^{L}\right),\left(a_{1}^{U}, a_{2}^{U}, a_{3}^{U}, a_{4}^{U} ; h_{A}^{U}\right)\right]$ and $\tilde{B}=\left[\tilde{B}^{L}, \tilde{B}^{U}\right]=\left[\left(b_{1}^{L}, b_{2}^{L}, b_{3}^{L}, b_{4}^{L} ; h_{B}^{L}\right),\left(b_{1}^{U}, b_{2}^{U}, b_{3}^{U}, b_{4}^{U} ; h_{B}^{U}\right)\right]$ be two non-negative IT2TrFSs; then arithmetic operations on these are defined as follows.

1. Addition: $A \oplus B=\left[\left(a_{1}^{L}+b_{1}^{L}, a_{2}^{L}+b_{2}^{L}, a_{3}^{L}+b_{3}^{L}, a_{4}^{L}+b_{4}^{L}\right.\right.$; $\left.\min \left(h_{A}^{L}, h_{B}^{L}\right)\right),\left(a_{1}^{U}+b_{1}^{U}, a_{2}^{U}+b_{2}^{U}, a_{3}^{U}+b_{3}^{U}, a_{4}^{U}+b_{4}^{U} ; \min \right.$ $\left.\left.\left(h_{A}^{U}, h_{B}^{U}\right)\right)\right]$.

2. Subtraction: $\quad A \ominus B=\left[\left(a_{1}^{L}-b_{4}^{L}, a_{2}^{L}-b_{3}^{L}, a_{3}^{L}-b_{2}^{L}, a_{4}^{L}-\right.\right.$ $\left.b_{1}^{L} ; \min \left(h_{A}^{L}, h_{B}^{L}\right)\right),\left(a_{1}^{U}-b_{4}^{U}, a_{2}^{U}-b_{3}^{U}, a_{3}^{U}-b_{2}^{U}, a_{4}^{U}-b_{1}^{U}\right.$; $\left.\left.\min \left(h_{A}^{U}, h_{B}^{U}\right)\right)\right]$.

3. Multiplication: $\quad A \otimes B=\left[\left(a_{1}^{L} \times b_{1}^{L}, a_{2}^{L} \times b_{2}^{L}, a_{3}^{L} \times\right.\right.$ $\left.b_{3}^{L}, a_{4}^{L} \times b_{4}^{L} ; \min \quad\left(h_{A}^{L}, h_{B}^{L}\right)\right),\left(a_{1}^{U} \times b_{1}^{U}, a_{2}^{U} \times b_{2}^{U}, a_{3}^{U} \times\right.$ $\left.\left.b_{3}^{U}, a_{4}^{U} \times b_{4}^{U} ; \min \left(h_{A}^{U}, h_{B}^{U}\right)\right)\right]$.

4. Division: $A \varnothing B=\left[\left(a_{1}^{L} / b_{4}^{L}, a_{2}^{L} / b_{3}^{L}, a_{3}^{L} / b_{2}^{L}, a_{4}^{L} / b_{1}^{L} ; \min \left(h_{A}^{L}\right.\right.\right.$, $\left.\left.\left.h_{B}^{L}\right)\right),\left(a_{1}^{U} / b_{4}^{U}, a_{2}^{U} / b_{3}^{U}, a_{3}^{U} / b_{2}^{U}, a_{4}^{U} / b_{1}^{U} ; \quad \min \left(h_{A}^{U}, h_{B}^{U}\right)\right)\right]$, where $b_{1}^{L}, b_{2}^{L}, b_{3}^{L}, b_{4}^{L}, b_{1}^{U}, b_{2}^{U}, b_{3}^{U}$ and $b_{4}^{U}$ are positive real numbers.

5. Multiplication by a real number $a$ :

$$
a \tilde{A}=\left\{\begin{array}{c}
{\left[\left(a a_{1}^{L}, a a_{2}^{L}, a a_{3}^{L}, a a_{4}^{L} ; h_{A}^{L}\right),\left(a a_{1}^{U}, a a_{2}^{U}, a a_{3}^{U}, a a_{4}^{U} ;\right.\right.} \\
\left.\left.h_{A}^{U}\right)\right], \text { if } a \geq 0 ; \\
{\left[\left(a a_{4}^{L}, a a_{3}^{L}, a a_{2}^{L}, a a_{1}^{L} ; h_{A}^{L}\right),\left(a a_{4}^{U}, a a_{3}^{U}, a a_{2}^{U}, a a_{1}^{U} ;\right.\right.} \\
\left.\left.h_{A}^{U}\right)\right], \text { if } a \leq 0 .
\end{array}\right.
$$

6. Division by a real number $a$ :

$$
\tilde{A} / a=\left\{\begin{array}{c}
{\left[\left(a_{1}^{L} / a, a_{2}^{L} / a, a_{3}^{L} / a, a_{4}^{L} / a ; h_{A}^{L}\right),\left(a_{1}^{U} / a, a_{2}^{U} / a\right.\right.} \\
\left.\left.a_{3}^{U} / a, a_{4}^{U} / a ; h_{A}^{U}\right)\right], \text { if } a>0 \\
{\left[\left(a_{4}^{L} / a, a_{3}^{L} / a, a_{2}^{L} / a, a_{1}^{L} / a ; h_{A}^{L}\right),\left(a_{4}^{U} / a, a_{3}^{U} / a\right.\right.} \\
\left.\left.a_{2}^{U} / a, a_{1}^{U} / a ; h_{A}^{U}\right)\right], \text { if } a<0 .
\end{array}\right.
$$

2.1d $\alpha$-cut of IT2TrFS [35]: If $\tilde{A}=\left[\tilde{A}^{L}, \tilde{A}^{U}\right]=\left[\left(a_{1}^{L}\right.\right.$, $\left.\left.a_{2}^{L}, a_{3}^{L}, a_{4}^{L} ; h_{A}^{L}\right),\left(a_{1}^{U}, a_{2}^{U}, a_{3}^{U}, a_{4}^{U} ; h_{A}^{U}\right)\right]$ is an IT2TrFS, then $\alpha$ cut of $\tilde{A}$, where $0 \leq \alpha \leq 1$, is defined as follows:

$$
\begin{aligned}
A_{\alpha} & =\left[A_{\alpha}^{L}, A_{\alpha}^{U}\right] \\
& =\left\{\begin{array}{cc}
{\left[\left[{ }^{l} A_{\alpha}^{L},{ }^{r} A_{\alpha}^{L}\right],\left[{ }^{l} A_{\alpha}^{U},{ }^{r} A_{\alpha}^{U}\right]\right],} & 0 \leq \alpha<h_{A}^{L} ; \\
{\left[{ }^{l} A_{\alpha}^{U},{ }^{r} A_{\alpha}^{U}\right],} & h_{A}^{L} \leq \alpha \leq h_{A}^{U} ;
\end{array}\right.
\end{aligned}
$$

where ${ }^{l} A_{\alpha}^{L}=a_{1}^{L}+\left(a_{2}^{L}-a_{1}^{L}\right) \alpha / h_{A}^{L}, \quad{ }^{r} A_{\alpha}^{L}=a_{4}^{L}-\left(a_{4}^{L}-a_{3}^{L}\right)$ $\alpha / h_{A}^{L},{ }^{l} A_{\alpha}^{U}=a_{1}^{U}+\left(a_{2}^{U}-a_{1}^{U}\right) \alpha / h_{A}^{U}$ and ${ }^{r} A_{\alpha}^{U}=a_{4}^{U}-\left(a_{4}^{U}\right.$ $\left.-a_{3}^{U}\right) \alpha / h_{A}^{U}$.

2.1e Signed distance of IT2TrFS: The signed distance method based on decomposition principle for a fuzzy number (type-1 fuzzy set, T1FS) is proposed by Yao and $\mathrm{Wu}$ [37]. Chen [35] extended that method for IT2TrFS. Let $\tilde{A}=\left[\tilde{A}^{L}, \tilde{A}^{U}\right]=\left[\left(a_{1}^{L}, a_{2}^{L}, a_{3}^{L}, a_{4}^{L} ; h_{A}^{L}\right),\left(a_{1}^{U}, a_{2}^{U}, a_{3}^{U}, a_{4}^{U} ; h_{A}^{U}\right)\right]$ be an IT2TrFS as defined earlier; then signed distance of $\tilde{A}$ is

$$
\begin{aligned}
d\left(\tilde{A}, 0_{1}\right)= & \frac{1}{8}\left[a_{1}^{L}+a_{2}^{L}+a_{3}^{L}+a_{4}^{L}+4 a_{1}^{U}+2 a_{2}^{U}+2 a_{3}^{U}+4 a_{4}^{U}\right. \\
& \left.+3\left(a_{2}^{U}+a_{3}^{U}-a_{1}^{U}-a_{4}^{U}\right) \frac{h_{A}^{L}}{h_{A}^{U}} .\right]
\end{aligned}
$$

If we take $a_{1}^{L}=a_{2}^{L}=a_{3}^{L}=a_{4}^{L}=a_{1}^{U}=a_{2}^{U}=a_{3}^{U}=a_{4}^{U}=a$, then IT2TrFS $\tilde{A}$ becomes a scaler point as $\tilde{A}=a$. From Eq. (2.4), $d\left(a, 0_{1}\right)=2 a$, i.e., signed distance of a real number $a$ from 0 is $2 a$. Now, we propose a de-fuzzification method of IT2TrFS, which is much easier from computational point of view and also overwhelms the afore-mentioned drawback.

\subsection{Interval approximation of IT2TrFS}

Definition The interval approximation of IT2TrFS $\tilde{A}$ is defined as follows:

$$
\begin{aligned}
{\left[A_{\alpha}^{-}, A_{\alpha}^{+}\right] } & =\left[\frac{1}{2} \int_{0}^{1}\left({ }^{l} A_{\alpha}^{L}+{ }^{l} A_{\alpha}^{U}\right) d \alpha, \frac{1}{2} \int_{0}^{1}\left({ }^{r} A_{\alpha}^{L}+{ }^{r} A_{\alpha}^{U}\right) d \alpha\right] \\
& =\left[\frac{1}{4}\left[\left(a_{1}^{L}+a_{2}^{L}\right) h_{A}^{L}+\left(a_{1}^{U}+a_{2}^{U}\right) h_{A}^{U}\right],\right. \\
& \left.\frac{1}{4}\left[\left(a_{3}^{L}+a_{4}^{L}\right) h_{A}^{L}+\left(a_{3}^{U}+a_{4}^{U}\right) h_{A}^{U}\right]\right] .
\end{aligned}
$$

Definition Mean (de-fuzzified to a scalar) value of the interval approximation of IT2TrFS $\tilde{A}$ is defined as

$$
\begin{aligned}
M(\tilde{A})= & \frac{A_{\alpha}^{-}+A_{\alpha}^{+}}{2} \\
= & \frac{1}{8}\left[\left(a_{1}^{L}+a_{2}^{L}+a_{3}^{L}+a_{4}^{L}\right) h_{A}^{L}\right. \\
& \left.+\left(a_{1}^{U}+a_{2}^{U}+a_{3}^{U}+a_{4}^{U}\right) h_{A}^{U}\right] .
\end{aligned}
$$


If we take $a_{1}^{L}=a_{2}^{L}=a_{3}^{L}=a_{4}^{L}=a_{1}^{U}=a_{2}^{U}=a_{3}^{U}=$ $a_{4}^{U}=a$, then from Eq. (2.6), $M(\tilde{A})=a\left(h_{A}^{L}+h_{A}^{U}\right) / 2$. Again if we take $h_{A}^{L}=h_{A}^{U}=1$ then $M(\tilde{A})=a$, i.e., if there is no fuzziness, then de-fuzzified value of IT2TrFS coincides with the crisp number.

\section{Notations and assumptions}

\section{Notations:}

$\lambda$ per year demand rate

$x$ per year screening rate

$p$ fraction of defective items, $0 \leq p<1$

$c$ per unit purchasing cost

$s$ per unit selling price

$v$ salvage value of per unit defective item

$h \quad$ holding cost per unit per year

$b$ backorder cost per unit per year

$d$ per unit screening cost

$K$ ordering cost per order

$y$ order quantity

$B$ backlogging quantity

$t$ time interval during which all items are screened

$T$ time interval between two replenishments

\section{Assumptions:}

1. A single type of product is considered and is instantly replenished, that is, lead time is zero.

2. Each lot of size $y$ contains a fraction $p$ of defective items, and defective items $p y$ are sold in a single batch at a discounted price prior to receiving next shipment.

3 . The screening process and demand proceed simultaneously, but screening rate is greater than demand rate (i.e., $x>\lambda$ ).

4. Shortage is allowed and is completely backlogged.

5. For avoiding the shortage during screening period $t$, perfect quality of items $(1-p) y$ must be greater than or equal to $\lambda t[5]$, that is,

$$
q \geq \frac{\lambda}{x}
$$

where $q=1-p$.

6. Although $100 \%$ screening process is not accomplished, yet shortage is backlogged without any defect [11].

\section{Mathematical formulation}

Figure 1 delineates the inventory level of item over time. At the beginning of the cycle a lot of size $y$ is replenished. After meeting up the shortage quantity $B$ of previous cycle, remaining items are stocked to fulfill the demand of instant cycle. Screening process and demand fulfillment occur simultaneously. However, $100 \%$ screening process is accomplished after time period $t$; it is assumed that the backorder quantity (of previous cycle) is delivered without any defects. After finishing the screening process, all imperfect quality items $p y$ are instantly withdrawn from the system. The remaining items are consumed at the rate $\lambda$, and then at the same rate, shortage occurs and continues up to time $T$. After this the cycle repeats itself till infinite time horizon.

The time interval $T$ between two replenishments is

$$
T=\frac{q y}{\lambda} .
$$

Total revenue per cycle is

$$
T R=s q y+v(1-q) y .
$$

The total cost per cycle (see, [6]) comprises with costs of ordering, purchasing, screening, holding, backordering and screening, which is expressed as follows:

$$
T C=K+c y+d y+h\left[\frac{(q y-B)^{2}}{2 \lambda}+\frac{(1-q) y^{2}}{x}\right]+\frac{b B^{2}}{2 \lambda} .
$$

Using Eqs. (4.1)-(4.3), the total average annual profit can be calculated as follows:

$$
\begin{aligned}
\operatorname{TPU}(y, B) & =\frac{T R-T C}{T}, \\
\operatorname{TPU}(y, B) & =\frac{\lambda}{q y}[s q y+v(1-q) y-K-c y-d y \\
& \left.-h\left\{\frac{(q y-B)^{2}}{2 \lambda}+\frac{(1-q) y^{2}}{x}\right\}-\frac{b B^{2}}{2 \lambda}\right] \\
& =h B+\left[\frac{h y}{x}+s-v\right] \lambda-\frac{h y}{2} q-\frac{(b+h) B^{2}}{2 y} \frac{1}{q} \\
& -\left[c-v+d+\frac{K}{y}+\frac{h y}{x}\right] \frac{\lambda}{q} .
\end{aligned}
$$

As discussed in the introduction section, demand forecasting process depends upon several uncertain, perturbing and unreliable sources/activities. Consequently, inherent impreciseness and vagueness do not describe a precise value of the demand. We can efficiently express it in terms of a fuzzy variable. Furthermore, if there are several experts and each expert's opinion is a fuzzy set, it differs from expert to expert. In such a situation, grade of membership of a particular value of demand is itself a fuzzy set in the interval $[0,1]$. That is, fuzziness may occur in grade of membership value itself. Hence, consideration of fuzzy demand (as in [17]) is not an efficacious task. We now consider that the final estimation of demand is a type-2 fuzzy set. Similarly, the fraction of imperfect quality items is also a type-2 fuzzy set. Let us assume that demand and fraction of good (non-defective) quality items (i.e., 


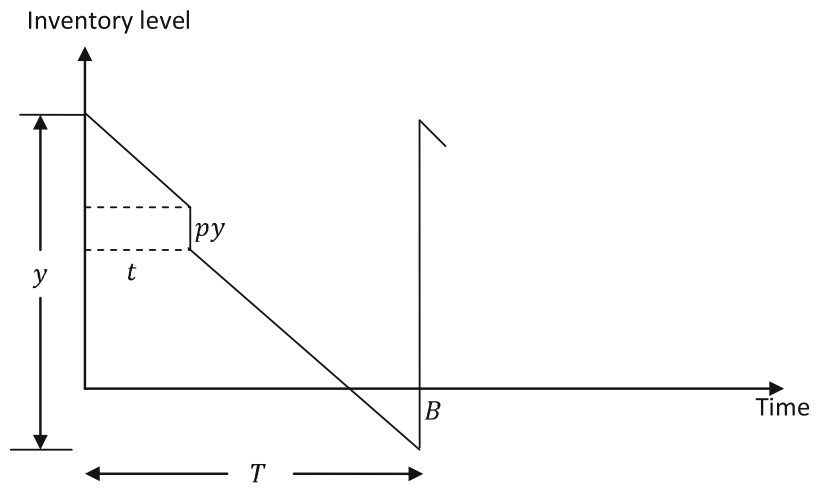

Figure 1. Inventory position over time.

$q=1-p)$ are IT2TrFSs, whose grades of membership are shown in figures 2 and 3, respectively, and are defined as follows: $\quad \tilde{\lambda}=\left[\tilde{\lambda}^{L}, \tilde{\lambda}^{U}\right]=\left[\left(\lambda_{1}^{L}, \lambda_{2}^{L}, \lambda_{3}^{L}, \lambda_{4}^{L} ; h_{\lambda}^{L}\right), \quad\left(\lambda_{1}^{U}, \lambda_{2}^{U}\right.\right.$, $\left.\left.\lambda_{3}^{U}, \lambda_{4}^{U} ; h_{\lambda}^{U}\right)\right] \quad$ and $\quad \tilde{q}=\left[\tilde{q}^{L}, \tilde{q}^{U}\right]=\left[\left(q_{1}^{L}, q_{2}^{L}, q_{3}^{L}, q_{4}^{L} ; h_{q}^{L}\right)\right.$, $\left.\left(q_{1}^{U}, q_{2}^{U}, q_{3}^{U}, q_{4}^{U} ; h_{q}^{U}\right)\right]$, where $\quad \lambda_{i}^{L} \leq \lambda_{i+1}^{L}, \lambda_{i}^{U} \leq \lambda_{i+1}^{U}$ $q_{i}^{L} \leq q_{i+1}^{L}, q_{i}^{U} \leq q_{i+1}^{U} ; i=1,2,3, \quad q_{1}^{U} \leq q_{1}^{L}, q_{4}^{L} \leq q_{4}^{U}, \lambda_{1}^{U}$ $\leq \lambda_{1}^{L}, \quad \lambda_{4}^{L} \leq \lambda_{4}^{U}, \quad 0 \leq q_{1}^{L}, q_{4}^{U}<1 \quad$ and $\quad 0<h_{q}^{L} \leq h_{q}^{U} \leq 1,0$ $<h_{\lambda}^{L} \leq h_{\lambda}^{U} \leq 1$. Arithmetic operations defined in section 2.2 are used here to calculate the fuzzy total profit per unit time as follows:

$$
\begin{aligned}
\widetilde{T P U}(y, B) & =h B+\left[\frac{h y}{x}+s-v\right] \tilde{\lambda} \ominus \frac{h y}{2} \tilde{q} \ominus \frac{(b+h) B^{2}}{2 y} \varnothing \tilde{q} \\
& \ominus\left[c-v+d+\frac{K}{y}+\frac{h y}{x}\right] \tilde{\lambda} \varnothing \tilde{q} .
\end{aligned}
$$

Depending upon the values of $h_{q}^{L}, h_{q}^{U}, h_{\lambda}^{L}$ and $h_{\lambda}^{U}$, four cases arise: (1) $h_{\lambda}^{L} \leq h_{q}^{L} \leq h_{\lambda}^{U} \leq h_{q}^{U}$, (2) $h_{\lambda}^{L} \leq h_{q}^{L} \leq h_{q}^{U} \leq h_{\lambda}^{U}$, (3) $h_{q}^{L} \leq h_{\lambda}^{L} \leq h_{q}^{U} \leq h_{\lambda}^{U}$ and (4) $h_{q}^{L} \leq h_{\lambda}^{L} \leq h_{\lambda}^{U} \leq h_{q}^{U}$. Hence the IT2TrFS profit function $\widetilde{T P U}$ can be expressed as

$$
\widetilde{T P U}=\left\{\begin{array}{c}
{\left[\left(T P U_{1}^{L}, T P U_{2}^{L}, T P U_{3}^{L}, T P U_{4}^{L} ; h_{\lambda}^{L}\right),\left(T P U_{1}^{U}, T P U_{2}^{U},\right.\right.} \\
\left.\left.T P U_{3}^{U}, T P U_{4}^{U}\right) ; h_{\lambda}^{U}\right], \quad h_{\lambda}^{L} \leq h_{q}^{L} \leq h_{\lambda}^{U} \leq h_{q}^{U} ; \\
{\left[\left(T P U_{1}^{L}, T P U_{2}^{L}, T P U_{3}^{L}, T P U_{4}^{L} ; h_{\lambda}^{L}\right),\left(T P U_{1}^{U}, T P U_{2}^{U},\right.\right.} \\
\left.\left.T P U_{3}^{U}, T P U_{4}^{U}\right) ; h_{q}^{U}\right], \quad h_{\lambda}^{L} \leq h_{q}^{L} \leq h_{q}^{U} \leq h_{\lambda}^{U} ; \\
{\left[\left(T P U_{1}^{L}, T P U_{2}^{L}, T P U_{3}^{L}, T P U_{4}^{L} ; h_{q}^{L}\right),\left(T P U_{1}^{U}, T P U_{2}^{U},\right.\right.} \\
\left.\left.T P U_{3}^{U}, T P U_{4}^{U}\right) ; h_{q}^{U}\right], \quad h_{q}^{L} \leq h_{\lambda}^{L} \leq h_{q}^{U} \leq h_{\lambda}^{U} ; \\
{\left[\left(T P U_{1}^{L}, T P U_{2}^{L}, T P U_{3}^{L}, T P U_{4}^{L} ; h_{q}^{L}\right),\left(T P U_{1}^{U}, T P U_{2}^{U},\right.\right.} \\
\left.\left.T P U_{3}^{U}, T P U_{4}^{U}\right) ; h_{\lambda}^{U}\right], h_{q}^{L} \leq h_{\lambda}^{L} \leq h_{\lambda}^{U} \leq h_{q}^{U}
\end{array}\right.
$$

with the condition

$$
\tilde{q}>\frac{\tilde{\lambda}}{x}[\text { Eq. (3.1) in IT2TrFS sense], }
$$

where

$$
\begin{aligned}
T P U_{i}^{L} & =h B+\left[\frac{h y}{x}+s-v\right] \lambda_{i}^{L}-\frac{h y}{2} q_{5-i}^{L}-\frac{(b+h) B^{2}}{2 y} \frac{1}{q_{i}^{L}} \\
& -\left[c-v+d+\frac{K}{y}+\frac{h y}{x}\right] \frac{\lambda_{5-i}^{L}}{q_{i}^{L}} ; i=1, \ldots, 4 .
\end{aligned}
$$

and

$$
\begin{aligned}
T P U_{i}^{U} & =h B+\left[\frac{h y}{x}+s-v\right] \lambda_{i}^{U}-\frac{h y}{2} q_{5-i}^{U}-\frac{(b+h) B^{2}}{2 y} \frac{1}{q_{i}^{U}} \\
& -\left[c-v+d+\frac{K}{y}+\frac{h y}{x}\right] \frac{\lambda_{5-i}^{U}}{q_{i}^{U}} ; i=1, \ldots, 4 .
\end{aligned}
$$

The de-fuzzified value of IT2TrFS profit function $\widetilde{T P U}$ given in Eq. (4.6) is as follows:

$$
M(\widetilde{T P U})=\left\{\begin{array}{l}
M\left(\widetilde{T P U} h_{\lambda}^{L}, h_{\lambda}^{U}\right), h_{\lambda}^{L} \leq h_{q}^{L} \leq h_{\lambda}^{U} \leq h_{q}^{U} \\
M\left(\widetilde{T P U} h_{\lambda}^{L}, h_{q}^{U}\right), h_{\lambda}^{L} \leq h_{q}^{L} \leq h_{q}^{U} \leq h_{\lambda}^{U} \\
M\left(\widetilde{T P U} h_{q}^{L}, h_{q}^{U}\right), h_{q}^{L} \leq h_{\lambda}^{L} \leq h_{q}^{U} \leq h_{\lambda}^{U} \\
M\left(\widetilde{T P U} h_{q}^{L}, h_{\lambda}^{U}\right), h_{q}^{L} \leq h_{\lambda}^{L} \leq h_{\lambda}^{U} \leq h_{q}^{U}
\end{array}\right.
$$

where for $m=h_{\lambda}^{L}, h_{q}^{L} ; n=h_{\lambda}^{U}, h_{q}^{U}$

$$
\begin{aligned}
M\left(\widetilde{T P U}_{m, n}\right)= & h B+\frac{1}{8}\left[( \frac { h y } { x } + s - v ) \left(\left(\lambda_{1}^{L}+\lambda_{2}^{L}+\lambda_{3}^{L}+\lambda_{4}^{L}\right) m\right.\right. \\
& \left.+\left(\lambda_{1}^{U}+\lambda_{2}^{U}+\lambda_{3}^{U}+\lambda_{4}^{U}\right) n\right)-\frac{h y}{2}\left(\left(q_{4}^{L}+q_{3}^{L}\right.\right. \\
& \left.\left.+q_{2}^{L}+q_{1}^{L}\right) m+\left(q_{4}^{U}+q_{3}^{U}+q_{2}^{U}+q_{1}^{U}\right) n\right) \\
& -\frac{(b+h) B^{2}}{2 y}\left(\left(\frac{1}{q_{1}^{L}}+\frac{1}{q_{2}^{L}}+\frac{1}{q_{3}^{L}}+\frac{1}{q_{4}^{L}}\right) m\right. \\
& \left.+\left(\frac{1}{q_{1}^{U}}+\frac{1}{q_{2}^{U}}+\frac{1}{q_{3}^{U}}+\frac{1}{q_{4}^{U}}\right) n\right) \\
& -\left(c-v+d+\frac{K}{y}+\frac{h y}{x}\right)\left(\left(\frac{\lambda_{1}^{L}}{q_{4}^{L}}+\frac{\lambda_{2}^{L}}{q_{3}^{L}}+\frac{\lambda_{3}^{L}}{q_{2}^{L}}\right.\right. \\
& \left.\left.\left.+\frac{\lambda_{4}^{L}}{q_{1}^{L}}\right) m+\left(\frac{\lambda_{1}^{U}}{q_{4}^{U}}+\frac{\lambda_{2}^{U}}{q_{3}^{U}}+\frac{\lambda_{3}^{U}}{q_{2}^{U}}+\frac{\lambda_{4}^{U}}{q_{1}^{U}}\right) n\right)\right] .
\end{aligned}
$$

The condition given in Eq. (4.7), becomes 


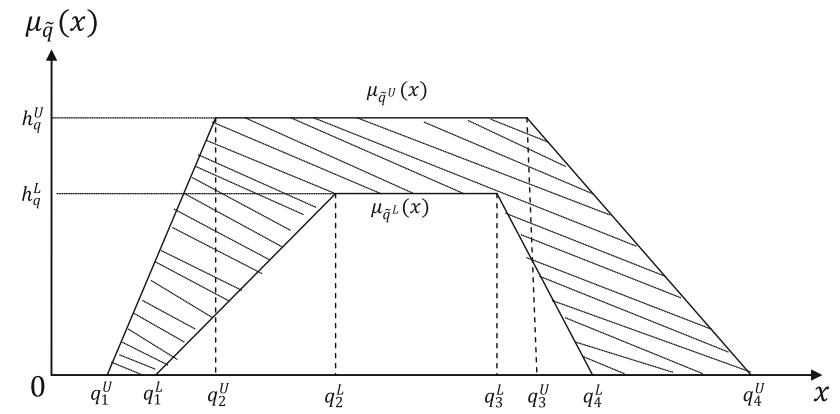

Figure 2. IT2TrFS $\tilde{q}$.

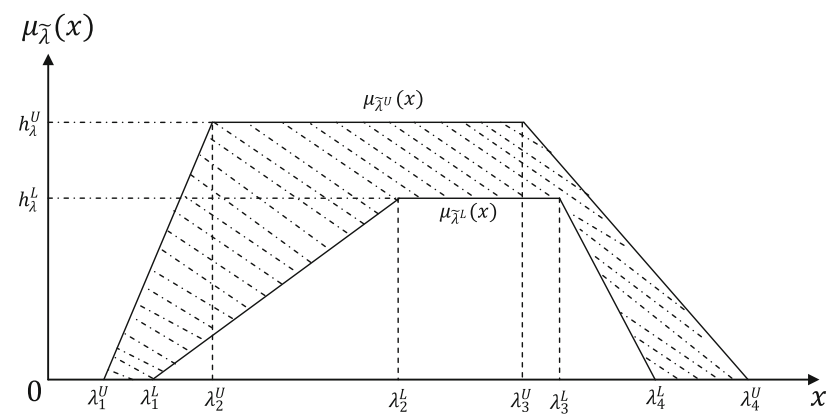

Figure 3. IT 2 TrFS demand.

$$
\begin{aligned}
& \left(\left(q_{1}^{L}+q_{2}^{L}+q_{3}^{L}+q_{4}^{L}\right) m+\left(q_{1}^{U}+q_{2}^{U}+q_{3}^{U}+q_{4}^{U}\right) n\right) \\
& \quad>\frac{1}{x}\left(\left(\lambda_{1}^{L}+\lambda_{2}^{L}+\lambda_{3}^{L}+\lambda_{4}^{L}\right) m+\left(\lambda_{1}^{U}+\lambda_{2}^{U}+\lambda_{3}^{U}+\lambda_{4}^{U}\right) n\right) \\
& \quad \text { for } m=h_{\lambda}^{L}, h_{q}^{L} ; n=h_{\lambda}^{U}, h_{q}^{U} .
\end{aligned}
$$

The profit function given in Eq. (4.11), can be written as follows:

$$
\begin{aligned}
M\left(\widetilde{T P U}_{m, n}\right) & =h B+\left(\frac{h y}{x}+s-v\right)\left(\lambda^{L} m+\lambda^{U} n\right) \\
& -\frac{h y}{2}\left(q^{L} m+q^{U} n\right)-\frac{(b+h) B^{2}}{2 y}\left(Q^{L} m+Q^{U} n\right) \\
& -\left(c-v+d+\frac{K}{y}+\frac{h y}{x}\right)\left(Z^{L} m+Z^{U} n\right)
\end{aligned}
$$

where

$$
\begin{aligned}
\lambda^{L} & =\frac{\lambda_{1}^{L}+\lambda_{2}^{L}+\lambda_{3}^{L}+\lambda_{4}^{L}}{8}, \lambda^{U}=\frac{\lambda_{1}^{U}+\lambda_{2}^{U}+\lambda_{3}^{U}+\lambda_{4}^{U}}{8} \\
q^{L} & =\frac{q_{1}^{L}+q_{2}^{L}+q_{3}^{L}+q_{4}^{L}}{8}, q^{U}=\frac{q_{1}^{U}+q_{2}^{U}+q_{3}^{U}+q_{4}^{U}}{8} \\
Q^{L} & =\frac{1}{8}\left(\frac{1}{q_{1}^{L}}+\frac{1}{q_{2}^{L}}+\frac{1}{q_{3}^{L}}+\frac{1}{q_{4}^{L}}\right), \\
Q^{U} & =\frac{1}{8}\left(\frac{1}{q_{1}^{U}}+\frac{1}{q_{2}^{U}}+\frac{1}{q_{3}^{U}}+\frac{1}{q_{4}^{U}}\right), \\
Z^{L} & =\frac{1}{8}\left(\frac{\lambda_{1}^{L}}{q_{4}^{L}}+\frac{\lambda_{2}^{L}}{q_{3}^{L}}+\frac{\lambda_{3}^{L}}{q_{2}^{L}}+\frac{\lambda_{4}^{L}}{q_{1}^{L}}\right), \\
Z^{U} & =\frac{1}{8}\left(\frac{\lambda_{1}^{U}}{q_{4}^{U}}+\frac{\lambda_{2}^{U}}{q_{3}^{U}}+\frac{\lambda_{3}^{U}}{q_{2}^{U}}+\frac{\lambda_{4}^{U}}{q_{1}^{U}}\right) .
\end{aligned}
$$

Theorem 1 The cost function $M\left(\widetilde{T P U}_{m, n}\right)$ is a concave function of $y$ and B. Moreover, the optimal policy is

$$
y_{m, n}^{*}=\sqrt{\frac{2 K\left(Z^{L} m+Z^{U} n\right)+(b+h) B^{2}\left(Q^{L} m+Q^{U} n\right)}{h\left[\left(Z^{L}-\lambda^{L}\right) \frac{2 m}{x}+\left(Z^{U}-\lambda^{U}\right) \frac{2 n}{x}+q^{L} m+q^{U} n\right]}}
$$

and

$$
B_{m, n}^{*}=\frac{h y_{m, n}^{*}}{(b+h)\left(Q^{L} m+Q^{U} n\right)} .
$$

Proof The first and the second order partial derivatives of $M\left(\widetilde{T P U}_{m, n}\right)$ are

$$
\begin{aligned}
& \frac{\partial M\left(\widetilde{T P U}_{m, n}\right)}{\partial B}=h-\frac{(b+h) B}{y}\left(Q^{L} m+Q^{U} n\right), \\
& \frac{\partial M\left(\widetilde{T P U}_{m, n}\right)}{\partial y}=\frac{h}{x}\left(\lambda^{L} m+\lambda^{U} n\right)-\frac{h}{2}\left(q^{L} m+q^{U} n\right) \\
&+\frac{(b+h) B^{2}}{2 y^{2}}\left(Q^{L} m+Q^{U} n\right) \\
&+\left(\frac{K}{y^{2}}-\frac{h}{x}\right)\left(Z^{L} m+Z^{U} n\right), \\
&\left.\frac{\partial^{2} M(\widetilde{T P U}}{\partial B^{2}}\right)=-\frac{(b+h)}{y}\left(Q^{L} m+Q^{U} n\right), \\
&\left.\frac{\partial^{2} M(\widetilde{T P U}}{\partial B \partial y}\right)=\frac{(b+h) B}{y^{2}}\left(Q^{L} m+Q^{U} n\right), \\
&\left.\frac{\partial^{2} M(\widetilde{T P U}}{\partial y^{2}}\right)=-\frac{(b+h) B^{2}}{y^{3}}\left(Q^{L} m+Q^{U} n\right) \\
& \frac{2 K}{y^{3}}\left(Z^{L} m+Z^{U} n\right) .
\end{aligned}
$$


Since $\quad \frac{\partial^{2} M\left(\widetilde{T P U} U_{m, n}\right)}{\partial B^{2}}<0, \quad \frac{\partial^{2} M\left(\widetilde{T P U} U_{m, n}\right)}{\partial B^{2}} \frac{\partial^{2} M\left(\widetilde{T P U} U_{m, n}\right)}{\partial y^{2}}$ $-\left[\frac{\partial^{2} M\left(\widetilde{T P U_{m, n}}\right)}{\partial B \partial y}\right]^{2}=\frac{2 K(b+h)}{y^{4}}\left(Q^{L} m Q^{U} n\right)\left(Z^{L} m+Z^{U} n\right)>0$.

Thus $M\left(\widetilde{T P U}_{m, n}\right)$ is a concave function of $y$ and $B$.

Now, the optimal order quantity $y_{m, n}^{*}$ and backorder quantity $B_{m, n}^{*}$ for $m=h_{\lambda}^{L}, h_{q}^{L} ; n=h_{\lambda}^{U}, h_{q}^{U}$ can be obtained from the relation $\partial M\left(\widetilde{T P U}_{m, n}\right) / \partial y=0$ $=\partial M\left(\widetilde{T P U}_{m, n}\right) / \partial B$.

$$
\begin{aligned}
& \frac{\partial M\left(\widetilde{T P U}_{m, n}\right)}{\partial y}=0 \Rightarrow \\
& y_{m, n}^{*}=\sqrt{\frac{2 K\left(Z^{L} m+Z^{U} n\right)+(b+h) B^{2}\left(Q^{L} m+Q^{U} n\right)}{h\left[\left(Z^{L}-\lambda^{L}\right) \frac{2 m}{x}+\left(Z^{U}-\lambda^{U}\right) \frac{2 n}{x}+q^{L} m+q^{U} n\right]}}
\end{aligned}
$$

and

$$
\frac{\partial M\left(\widetilde{T P U}_{m, n}\right)}{\partial B}=0 \Rightarrow B_{m, n}^{*}=\frac{h y_{m, n}^{*}}{(b+h)\left(Q^{L} m+Q^{U} n\right)}
$$

\section{Analysis}

If we put $\lambda_{1}^{L}=\lambda_{2}^{L}=\lambda_{3}^{L}=\lambda_{4}^{L}=\lambda_{1}^{U}=\lambda_{2}^{U}=\lambda_{3}^{U}=\lambda_{4}^{U}=\lambda$, $q_{1}^{L}=q_{2}^{L}=q_{3}^{L}=q_{4}^{L}=q_{1}^{U}=q_{2}^{U}=q_{3}^{U}=q_{4}^{U}=q \quad$ and $m=n=1$, then Theorem 1 gives

$$
\begin{gathered}
y^{*}=\sqrt{\frac{\left(2 K \lambda+b B^{2}+h B^{2}\right) \frac{1}{q}}{h\left(q-\frac{2 \lambda}{x}+\frac{2 \lambda}{x q}\right)},} \\
B^{*}=\frac{h y^{*}}{(b+h) \frac{1}{q}} .
\end{gathered}
$$

This is the optimal policy of Wee et al [6]. If shortage is not allowed in this model, i.e., $b \rightarrow \infty$, then $B^{*} \rightarrow 0$ and

$$
y^{*}=\sqrt{\frac{2 K \lambda / q}{h(q-2 \lambda / x+2 \lambda / x q)}} .
$$

This is the model of Salameh and Jaber [5].

\section{Illustrative example}

A shoe manufacturer company produces and supplies shoes to a retailer. The retailer knows that due to imperfect production processes and/or transportation, some shoes are partially damaged. Hence, he screens all the received shoes before selling, and defective shoes are also sold on discounted price. Fraction of defective shoes in the received lots varies from lot to lot, and is estimated as IT2TrFS.
Based on previous years demand pattern and experts' opinion, the estimated annual demand is also an IT2TrFS as given in table 1 . The cost parameters and other relevant information are also given in the table.

We are given that $h_{\lambda}^{L}=0.8, h_{\lambda}^{U}=1, h_{p}^{L}=0.9$ and $h_{p}^{U}=0.95 ; \quad \Rightarrow m=\min \left\{h_{\lambda}^{L}, h_{p}^{L}\right\}=0.8 \quad$ and $\quad n=$ $\min \left\{h_{\lambda}^{U}, h_{p}^{U}\right\}=0.95$. Hence, the optimal decision policy is $y^{*}=2030.74, B^{*}=695.132$ and $T P U^{*}=1117350$.

\subsection{Sensitivity analysis}

For given values of $\tilde{\lambda}$ and $\tilde{p}$, the sensitivity of heights, written in the form of $\left(h_{\lambda}^{L}, h_{\lambda}^{U}, h_{p}^{L}, h_{p}^{U}\right)$, is obtained in table 2. When $m$ or $n$ or both increase, $T P U_{m, n}^{*}$ is increased, while $y_{m, n}^{*}$ and $B_{m, n}^{*}$ are decreased. Hence, we can conclude that if fuzzy demand and fuzzy fraction of imperfect quality item are estimated with larger heights grade of membership, then profit is maximum.

Sensitivity of height of $\tilde{\lambda}$ for a crisp $p=0.07$ and sensitivity of height of $\tilde{p}$ for a crisp $\lambda=50000$ are examined in tables 3 and 4 , respectively. Table 3 shows that when $h_{\lambda}^{L}$ is increased and $h_{\lambda}^{U}$ is fixed, $T P U_{m, n}^{*}$ increases while $y_{m, n}^{*}$ and $B_{m, n}^{*}$ decrease. The same tendency can be observed when $h_{\lambda}^{U}$ is increased and $h_{\lambda}^{L}$ is fixed, as shown in table 4 .

Sensitivity of IT2Tr fuzzy demand for crisp $p=0.7$ and IT2TrFS $\tilde{p}$ for crisp $\lambda=50000$ are shown in tables 5 and 6 , respectively. Table 5 shows that when the parameter $\tilde{\lambda}$ is increased, $T P U_{m, n}^{*}, y_{m, n}^{*}$ and $B_{m, n}^{*}$ increase, because the defuzzified demand is increased. Table 6 shows that when the parameter $\tilde{p}$ is increased, $y_{m, n}^{*}$ increases while $T P U_{m, n}^{*}$ and $B_{m, n}^{*}$ decrease. This is because the de-fuzzified value of imperfect quality is increased due to increasing parameters' value of $\tilde{p}$. Consequently, the retailer procures more items and gains less profit.

Table 1. Costs and other parameters in appropriate units.

\begin{tabular}{lc}
\hline Notation & Co-efficient \\
\hline$\tilde{\lambda}$ & $((45000,50000,55000,60000 ; 0.8)$, \\
& $(40000,50000,60000,70000 ; 1))$ \\
$\tilde{p}$ & $((0.05,0.07,0.1,0.15 ; 0.9)$, \\
& $(0.02,0.07,0.12,0.2 ; 0.95))$ \\
$K$ & 100 \\
$h$ & 5 \\
$x$ & 175200 \\
$d$ & 0.5 \\
$c$ & 25 \\
$b$ & 10 \\
$s$ & 50 \\
$v$ & 20 \\
\hline
\end{tabular}


Table 2. Sensitivity of heights of IT2TrFSs $\tilde{\lambda}$ and $\tilde{p}$.

\begin{tabular}{|c|c|c|c|c|}
\hline$\left(h_{\lambda}^{L}, h_{\lambda}^{U}, h_{p}^{L}, h_{p}^{U}\right)$ & $(m, n)$ & $y_{m, n}^{*}$ & $B_{m, n}^{*}$ & $T P U_{m, n}^{*}$ \\
\hline$(0.8,0.8,1,1)$ & $(0.8,0.8)$ & 2176.38 & 815.34 & 1020240 \\
\hline$(0.8,0.85,0.95,1)$ & $(0.8,0.85)$ & 2119.68 & 769.87 & 1052600 \\
\hline$(0.85,0.85,0.95,1)$ & $(0.85,0.85)$ & 2070.51 & 730.06 & 1083700 \\
\hline$(0.8,0.95,0.85,0.9)$ & $(0.8,0.9)$ & 2071.76 & 730.18 & 1084970 \\
\hline$(0.9,0.95,0.85,0.9)$ & $(0.85,0.9)$ & 2029.49 & 695.00 & 1116080 \\
\hline$(0.95,0.95,0.9,0.95)$ & $(0.9,0.95)$ & 1961.83 & 635.53 & 1179580 \\
\hline$(1,1,1,1)$ & $(1,1)$ & 1886.73 & 565.47 & 1274240 \\
\hline
\end{tabular}

Table 3. Sensitivity of height of $\tilde{\lambda}$ when $p=0.07$.

\begin{tabular}{|c|c|c|c|}
\hline$\tilde{\lambda}$ & $y_{m, n}^{*}$ & $B_{m, n}^{*}$ & $T P U_{m, n}^{*}$ \\
\hline$((45000,50000,55000,60000 ; 0.80),(40000,50000,60000,70000 ; 0.90))$ & 2056.38 & 750.16 & 1097410 \\
\hline$((45000,50000,55000,60000 ; 0.85),(40000,50000,60000,70000 ; 0.90))$ & 2012.26 & 712.91 & 1128760 \\
\hline$((45000,50000,55000,60000 ; 0.90),(40000,50000,60000,70000 ; 0.90))$ & 1973.69 & 679.83 & 1160120 \\
\hline$((45000,50000,55000,60000 ; 0.80),(40000,50000,60000,70000 ; 0.85))$ & 2107.73 & 792.00 & 1064560 \\
\hline$((45000,50000,55000,60000 ; 0.80),(40000,50000,60000,70000 ; 0.95))$ & 2013.49 & 713.35 & 1130260 \\
\hline
\end{tabular}

Table 4. Sensitivity of height of $\tilde{p}$ when $\lambda=50000$.

\begin{tabular}{lccc}
\hline$\tilde{p}$ & $y_{m, n}^{*}$ & $B_{m, n}^{*}$ & 707.73 \\
$((0.05,0.07,0.10,0.15 ; 0.80),(0.02,0.07,0.12,0.20 ; 0.90))$ & 2008.06 & 673.53 & 1010540 \\
$((0.05,0.07,0.10,0.15 ; 0.85),(0.02,0.07,0.12,0.20 ; 0.90))$ & 1966.79 & 643.05 & 1040190 \\
$((0.05,0.07,0.10,0.15 ; 0.90),(0.02,0.07,0.12,0.20 ; 0.90))$ & 1931.04 & 746.78 & 1069850 \\
$((0.05,0.07,0.10,0.15 ; 0.80),(0.02,0.07,0.12,0.20 ; 0.85))$ & 2056.10 & 681004 \\
$((0.05,0.07,0.10,0.15 ; 0.80),(0.02,0.07,0.12,0.20 ; 0.95))$ & 1966.95 & 673.30 & 1040080 \\
\hline
\end{tabular}

Table 5. Sensitivity of $\tilde{\lambda}$ when $p=0.07$.

\begin{tabular}{|c|c|c|c|}
\hline$\tilde{\lambda}$ & $y_{m, n}^{*}$ & $B_{m, n}^{*}$ & $T P U_{m, n}^{*}$ \\
\hline$((40000,42000,45000,48000 ; 0.8),(36000,40000,45000,50000 ; 0.95))$ & 1818.07 & 644.17 & 906348 \\
\hline$((42000,45000,50000,55000 ; 0.8),(38000,45000,50000,60000 ; 0.95))$ & 1911.70 & 677.32 & 1009960 \\
\hline$((45000,50000,55000,60000 ; 0.8),(40000,50000,60000,70000 ; 0.95))$ & 2013.45 & 713.35 & 1130260 \\
\hline$((50000,55000,60000,65000 ; 0.8),(45000,55000,65000,75000 ; 0.95))$ & 2097.01 & 742.94 & 1235400 \\
\hline$((55000,60000,65000,70000 ; 0.8),(50000,60000,70000,80000 ; 0.95))$ & 2176.16 & 770.98 & 1340550 \\
\hline
\end{tabular}

\section{Conclusions}

In this paper, a more realistic EOQ model for items with imperfect quality is developed by considering type-2 fuzzy demand and type-2 fuzzy imperfect quality items, wherein shortages are allowed and are backlogged. We know that demand is one of the most important factors for any business, and is often predicted by previous data records and anticipation of forthcoming scenarios that include many uncertain events such as incomplete data set, vagueness in scenarios, imprecise nature of demand and so on. Hence, in order to capture such types of uncertainties, a more efficient technique, namely, interval type- 2 fuzzy demand rate is considered here. The paper also considered interval type- 2 fuzzy imperfect quality items, and proposed a method to de-fuzzify it into a crisp quantity. The proposed method is 
Table 6. Sensitivity of $\tilde{p}$ when $\lambda=50000$.

\begin{tabular}{|c|c|c|c|}
\hline$\tilde{p}$ & $y_{m, n}^{*}$ & $B_{m, n}^{*}$ & $T P U_{m, n}^{*}$ \\
\hline$((0.02,0.03,0.06,0.09 ; 0.9),(0.01,0.04,0.08,0.10 ; 0.95))$ & 1860.86 & 633.75 & 1113250 \\
\hline$((0.04,0.05,0.08,0.12 ; 0.9),(0.02,0.06,0.09,0.16 ; 0.95))$ & 1882.46 & 624.3 & 1105990 \\
\hline$((0.05,0.07,0.10,0.15 ; 0.9),(0.02,0.07,0.12,0.20 ; 0.95))$ & 1899.97 & 615.48 & 1099400 \\
\hline$((0.06,0.07,0.11,0.15 ; 0.9),(0.03,0.08,0.14,0.20 ; 0.95))$ & 1906.98 & 612.73 & 1097080 \\
\hline$((0.09,0.11,0.15,0.20 ; 0.9),(0.05,0.14,0.20,0.25 ; 0.95))$ & 1944.74 & 593.16 & 1081760 \\
\hline
\end{tabular}

applied to estimate the equivalent crisp total average profit function. Some existing models are derived from our model; hence, these models can be seen as special cases of this model. Numerical illustration is also presented to support the mathematical formulation and methodology. Finally, from the sensitivity analysis, it is observed that parameters of type- 2 fuzzy variables significantly influence the decision policy. This model can be further extended by exempting the assumption that backlog quantity is delivered without defect; furthermore, partial backlogging and fuzzy type- 2 cost parameters can be taken into account.

\section{Acknowledgements}

The author expresses sincere thanks to the editor, the associate editor and the anonymous reviewers for their valuable and constructive comments and suggestions, which have led to a significant improvement in an earlier version of the manuscript.

\section{References}

[1] Maiyar L M and Thakkar J J 2017 A combined tactical and operational deterministic food grain transportation model: particle swarm based optimization approach. Computers \& Industrial Engineering 110: 30-42

[2] Mogale D G, Kumar S K and Tiwari M K 2018 An MINLP model to support the movement and storage decisions of the Indian food grain supply chain. Control Engineering Practice 70: 98-113

[3] Shekarian E, Kazemi N, Rashid S H A and Olugu U E 2017 Fuzzy inventory models: a comprehensive review. Applied Soft Computing 55: 588-621

[4] Rosenblatt M J and Lee H L 1986 Economic production cycles with imperfect production processes. IIE Transactions 18(1): 48-55

[5] Salameh M K and Jaber M Y 2000 Economic production quantity model for items with imperfect quality. International Journal of Production Economics 64(1): 59-64

[6] Wee H M, Yu J and Chen M C 2007 Optimal inventory model for items with imperfect quality and shortage backordering. Omega 35(1): 7-11

[7] Eroglu A and Ozdemir G 2007 An economic order quantity model with defective items and shortages. International Journal of Production Economics 106(2): 544-549
[8] Maddah B and Jaber M Y 2008 Economic order quantity for items with imperfect quality: revisited. International Journal of Production Economics 112(2): 808-815

[9] Maddah B, Salameh M K and Moussawi-Haidar L 2010 Order overlapping: a practical approach for preventing shortages during screening. Computers \& Industrial Engineering 58(4): 691-695

[10] Bag S, Tiwari M K and Chan T 2017 Predicting the consumer's purchase intention of durable goods: an attributelevel analysis. Journal of Business Research https://doi.org/ 10.1016/j.jbusres.2017.11.031

[11] Chang H C and Ho C H 2010 Exact closed-form solutions for optimal inventory model for items with imperfect quality and shortage backordering. Omega 38(3): 233-237

[12] Wang W T, Wee H M, Cheng Y L, Wen C L and CárdenasBarrón L E 2015 EOQ model for imperfect quality items with partial backorders and screening constraint. European Journal of Industrial Engineering 9(6): 744-773

[13] Rezaei J 2016 Economic order quantity and sampling inspection plans for imperfect items. Computers \& Industrial Engineering 96: 1-7

[14] Taleizadeh A A, Khanbaglo M P S and Cárdenas-Barrón L E 2016 An EOQ inventory model with partial backordering and reparation of imperfect products. International Journal of Production Economics 182: 418-434

[15] Khan M, Hussain M and Cárdenas-Barrón L E 2017 Learning and screening errors in an EPQ inventory model for supply chains with stochastic lead time demands. International Journal of Production Research 55(16): 4816-4832

[16] Yao J S, Chang S C and Su J S 2000 Fuzzy inventory without backorder for fuzzy order quantity and fuzzy total demand quantity. Computers \& Operations Research 27(10): 935-962

[17] Chang H C 2004 An application of fuzzy sets theory to the EOQ model with imperfect quality items. Computers \& Operations Research 31(12): 2079-2092

[18] Björk K M 2009 An analytical solution to a fuzzy economic order quantity problem. International Journal of Approximate Reasoning 50(3): 485-493

[19] Liu J and Zheng H 2012 Fuzzy economic order quantity model with imperfect items, shortages and inspection errors. Systems Engineering Procedia 4: 282-289

[20] Kumar R S and Goswami A 2015 A continuous review production-inventory system in fuzzy random environment: minmax distribution free procedure. Computers \& Industrial Engineering 79: 65-75

[21] Kumar R S, Tiwari M K and Goswami A 2016 Two-echelon fuzzy stochastic supply chain for the manufacturer-buyer integrated production-inventory system. Journal of Intelligent Manufacturing 27(4): 875-888 
[22] Chang H C, Yao J S and Ouyang L Y 2006 Fuzzy mixture inventory model involving fuzzy random variable lead time demand and fuzzy total demand. European Journal of Operational Research 169(1): 65-80

[23] Lin Y J 2008 A periodic review inventory model involving fuzzy expected demand short and fuzzy backorder rate. Computers \& Industrial Engineering 54(3): 666-676

[24] Dutta P, Chakraborty D and Roy A R 2005 A single-period inventory model with fuzzy random variable demand. Mathematical and Computer Modelling 41(8-9): 915-922

[25] Dutta P, Chakraborty D and Roy A R 2007 Continuous review inventory model in mixed fuzzy and stochastic environment. Applied Mathematics and Computation 188(1): 970-980

[26] Dey O and Chakraborty D 2009 Fuzzy periodic review system with fuzzy random variable demand. European Journal of Operational Research 198(1): 113-120

[27] Dey O and Chakraborty D 2011 A fuzzy random continuous review inventory system. International Journal of Production Economics 132(1): 101-106

[28] Wang X 2011 Continuous review inventory model with variable lead time in a fuzzy random environment. Expert Systems with Applications 38(9): 11715-11721

[29] Kumar R S and Goswami A 2013 Fuzzy stochastic EOQ inventory model for items with imperfect quality and shortages are backlogged. Advanced Modeling and Optimization 15(2): 261-279
[30] Bhuiya S K and Chakraborty D 2016 A fuzzy random EPQ model with fuzzy defective rates and fuzzy inspection errors. Journal of Intelligent \& Fuzzy Systems 30(6): $3527-3541$

[31] Karnik N N and Mendel J M Centroid of a type-2 fuzzy set. Information Sciences 132(1): 195-220

[32] Wu D and Mendel J M 2007 Uncertainty measures for interval type-2 fuzzy sets. Information Sciences 177(23): 5378-5393

[33] Wu D and Mendel J M 2009 A comparative study of ranking methods, similarity measures and uncertainty measures for interval type-2 fuzzy sets. Information Sciences 179(8): 1169-1192

[34] Mendel J M and Wu H 2007 New results about the centroid of an interval type-2 fuzzy set, including the centroid of a fuzzy granule. Information Sciences 177(2): 360-377

[35] Chen T Y 2011 An integrated approach for assessing criterion importance with interval type- 2 fuzzy sets and signed distances. Journal of the Chinese Institute of Industrial Engineers 28(8): 553-572

[36] Mizumoto M and Tanaka K 1981 Fuzzy sets and type-2 under algebraic product and algebraic sum. Fuzzy Sets and Systems 5(3): 277-290

[37] Yao J S and Wu K 2000 Ranking fuzzy numbers based on decomposition principle and signed distance. Fuzzy Sets and Systems 116(2): 275-288 